\title{
Low-Temperature Conductivity of Silicon Doped with Antimony
}

\author{
A. K. Fedotov ${ }^{a *}$, I. A. Svito ${ }^{a}$, V. V. Fedotova ${ }^{b}$, A. G. Trafimenko ${ }^{c}$, A. L. Danilyuk ${ }^{c}$, and S. L. Prischepa \\ ${ }^{a}$ Belarusian State University, 220030 Minsk, Belarus \\ ${ }^{b}$ Scientific-Practical Materials Research Center, National Academy of Sciences of Belarus, 220078 Minsk, Belarus \\ ${ }^{c}$ Belarusian State University of Informatics and Radioelectronics, 220013 Minsk, Belarus \\ *e-mail:fedotov@bsu.by \\ Submitted September 4, 2014; accepted for publication October 22, 2014
}

\begin{abstract}
A detailed analysis of the experimental temperature dependences of the resistivity of silicon doped with arsenic with a concentration of $10^{18} \mathrm{~cm}^{-3}$ is performed for the region $1.8 \mathrm{~K}<T<25 \mathrm{~K}$. It is shown that, as a result of cooling to a temperature lower than $4.5 \mathrm{~K}$, a transition from the Mott mode with variable hopping length to the mode of hopping conduction via nearest neighbors is observed, while, at $T<2.5 \mathrm{~K}$, a transition to the Shklovskii-Efros mechanism is possible. A model for such a temperature crossover is suggested; the model is based on simplified solution of the percolation problem with the use of an interpolation expression for the density of states. Performed estimates show that the model is in satisfactory agreement with experimental data when the minimum number of adjustable parameters are used.
\end{abstract}

DOI: $10.1134 / \mathrm{S} 1063782615060093$

\section{INTRODUCTION}

At present, there are no signs of the lessening of interest in the study of mechanisms of conduction in semiconducting materials at low temperatures, including such materials as traditional heavily doped semiconductors in the vicinity of the metal-insulator transition $[1,2]$. This is related both to fundamental problems of electron transport in the vicinity of these transitions and to some applied aspects. In particular, the features of the mechanisms of low-temperature electrical conductivity in doped semiconductors with the involvement of multicharged localized states, the mechanisms of the manifestation of positive and negative magnetoresistance (MR), the trends in the localization and specific features of the energy structure of bands for impurity and localized states, and so on, remain not quite clearly understood. In this context, detailed study of conduction in heavily doped semiconductors within a wide temperature range under the effect of a magnetic field remains pressing.

The behavior of the dependence of the specific resistance on temperature and magnetic-field induction $\rho(T, B)$ in heavily doped semiconductors in the case where the metal-insulator is approached from the semiconductor side has been the subject of many experimental and theoretical studies over the last 20 years. The metal-insulator transition takes place when the Mott criterion $N_{C}^{1 / 3} a_{\mathrm{B}} \approx 0.25$ becomes satisfied (here, $N_{C}$ is the critical concentration of impurity centers and $a_{\mathrm{B}}$ is the effective Bohr radius of an isolated center). This criterion was verified in various experiments [4, 5]. Nevertheless, there still remain some problems with correct understanding of the temperature dependences of the specific resistance and MR in the vicinity of the critical concentration of localized centers, i.e., in the situation where, at low temperatures, competition between hopping conduction (with a constant or variable hop length) of various types, mechanisms of weak localization, and percolation-related or band-type (metallic and/or impuritybased) conduction typically takes place. Thus, a number of crossovers between different mechanisms can be observed in a wide range of temperatures; this makes it necessary take into account these crossovers very accurately in order to obtain the correct values of model characteristic parameters from the experimental dependences $\rho(T, B)$.

\section{MAIN APPROACHES TO THE DESCRIPTION OF CROSSOVERS}

In available publications, we can find quite a number of models of crossovers between hopping conduction in accordance with the Mott and Shklovskii-Efros mechanisms for variable-range hopping (the VRH mode) [6-12]. The temperature dependences of the specific resistance for these mechanisms are described by the well-known expression

$$
\rho(T)=\rho_{0} \exp \left(T^{*} / T\right)^{p},
$$

where $\rho_{0}$ is the pre-exponential factor, while $T^{*}$ and $p$ are the characteristic temperature and the exponent, which are defined by the mechanism of hopping con- 
duction (by the character of the energy dependence of the electron density of states near the Fermi energy). In the three-dimensional case, expression (1) characterizes the VRH mode $[3,5]$ with characteristic temperatures $T^{*}=T_{M}=\beta_{0} / k_{\mathrm{B}} g_{0} a^{3}$ for the Mott mechanism and the exponent $p=1 / 4$ and, correspondingly, $T^{*}=T_{0}=\beta_{1} q^{2} /\left(k_{\mathrm{B}} a \kappa\right)$ at $p=1 / 2$ for the ShklovskiiEfros mechanism.

On the other hand, there are a small number of publications concerned with crossovers from the VRH mechanisms to the mode of hopping conduction with hops via the nearest states in the Coulomb gap (nearest-neighbor hopping, the NNH mode), in which case $T^{*}=T_{N}=E_{N} / k_{\mathrm{B}}$ and $p=1$ [13-15]. We introduced the following designations in the above relations for $T^{*}: \beta_{0}=21.2$ and $\beta_{1}=2.8$ are numerical coefficients; $a$ is the localization radius of an electron, $\kappa$ is the permittivity (absolute) of the material, $g_{0}$ is the density of states at the Fermi level (for the Mott mechanism, $g_{0}=$ const, i.e., this parameter is independent of energy), $k_{\mathrm{B}}$ is the Boltzmann constant, $q$ is the elementary charge, and $E_{N}$ is the activation energy for conduction according to nearest-neighbor hopping.

It follows from relation (1) that the characteristics of the VRH and NNH modes of conduction differ both in terms of the numerical parameters involved in $T^{*}$ and possibly in terms of the physical pattern itself. On the other hand, the temperature range of the crossover in this case is typically found to be fairly wide so that the correct comparison of theory with the experiment, as was mentioned above, can be noticeably complicated. Therefore, in order to make it possible to compare the experimental values of the parameters $T_{M}, T_{0}$, and $T_{N}$ with the calculated ones, it is important to correctly describe the crossover range both from the experimental and the theoretical (model) points of view. From the point of view of an experiment, the methodology of such a description is typically based on two approaches. In the first approach, researchers determine the temperature range where the dependences (1) are linearized in the Mott coordinates $\ln \rho-(1 / T)^{p}$ in the corresponding temperature ranges. This makes it possible to determine the value of $T^{*}$ (from the slope angle) and also the exponent $p$ (by exhaustively searching through known model values). In the second approach [16, 17], researchers search for the temperature region corresponding to linearization of the reduced activation energy $w(T)=-\partial \log \rho / \partial \log T$, which formally makes it possible to determine the exponent $p$ in relation (1). In both approaches, the correctness of the determination of $T^{*}$ and $p$ is appreciably affected by the following factors: the accuracy of temperature stabilization at the experimental points $\rho(T)$, the number of mechanisms in the crossover's temperature range under study, and the temperature dependence of pre-exponential factors $\rho_{0}$ in expressions of type (1). The two last factors, evidently, are especially important for samples with a localized-center concentration close to the metal-insulator transition. For such samples, the range of variation in the resistance in the temperature region $2-25 \mathrm{~K}$ under investigation can be fairly small and the number of mechanisms of conduction observed in this region, in contrast, can be large (from two to four). In the latter case, in order to derive the mechanisms involved in the crossover, additional experiments with measurements of the Hall effect and MR are required. It is worth noting that, for the samples, which are far from the Mott transition, the third factor can be apparently disregarded in a number of cases [18].

The known theoretical models of crossovers from the Mott mechanism to the Shklovskii-Efros mechanism are characterized by two different approaches to the determination of exponent $p$ in relation (1). The first approach is based on analysis of the percolation problem [6-10], while the second approach is based on optimization of the exponent in the expression for hopping conduction with the use of an interpolation expression for the density of states [11, 12]. The second approach is less strict than analysis of the percolation problem. As was shown by Meir [10], this approach leads to noticeable overestimation of the width of the crossover region.

Meir [10] suggested a general approach to description of the crossover from the Mott mechanism to the Shklovskii-Efros mechanism; this approach leads to a fairly complex multiparameter integral equation, which does not allow analytical processing and requires fairly complex numerical analysis.

In order to describe crossovers in the two- and three-dimensional situations, Van Lien et al. [11, 12, $14,15]$ suggested a procedure based on optimization of the exponent in the expression for the probability of hopping $(\sim \exp (-\eta)$, where $\eta=(2 r / a)+(E / k T), r$ is the hop length, and $E$ is the activation energy for a hop) using an interpolation expression for the density of states $g(E) \propto|E|^{n}$ ( $n$ is the integer exponent, which is equal to 0 for the Mott mechanism and to 2 for the Shklovskii-Efros mechanism). This approach leads to comparatively simple analytical expressions but is less rigorous than analysis of the percolation problem.

Agrinskaya and Kozub [9] suggested a relatively simple approach to description of the crossover from the Mott mechanism to the Shklovskii-Efros mechanism; this approach is based (as the approach suggested in [10]) on considerations of percolation theory; however, this approach provides simpler analysis of the connectivity condition. The theoretical equation for the critical value of the exponent $\xi(T)$ in the temperature dependence of the hopping resistance was obtained as

$$
\ln \rho(T)=A \xi(T)
$$


where $A$ is the coefficient and the relation between the function $\xi(T)$ and the exponent $p$ in (1) corresponds to the expression $\xi \propto\left(T^{*} / T\right)^{p}$.

As for the crossover from the VRH mode to the NNH mode, we know only one fairly substantiated model suggested by Rosenbaum [14, 15]; this model is based on the procedure of optimization of the exponent (the second approach above).

In this study, we undertake an attempt to provide a description of the experimentally observed crossover from the VRH mode to the NNH mode; the description is based on a simplified solution of the percolation problem with the use of an interpolation expression for the density of states; this solution is valid for both modes.

\section{EXPERIMENTAL}

The samples for electrical measurements were cut from commercial wafers of single-crystal silicon of the $n$-Si:Sb type $(\rho=0.01 \mathrm{Ohm} \mathrm{cm})$ with the orientation (100) in the form of strips with a thickness of $1 \mathrm{~mm}$ and a length of $12 \mathrm{~mm}$. Onto the rectangular samples two current contacts, two Hall contacts, and two potential contacts were deposited by means of ultrasonic soldering. The ohmic behavior of the contacts was controlled by measurements of the current-voltage $(I-V)$ characteristics. The $I-V$ characteristics were strictly linear in the entire range of temperatures under study.

In order to perform electrical measurements, the fabricated samples were soldered with the use of copper microwires in a special measurement probe, which was placed into a CHNF system (Cryogenics Ltd., England) based on a closed-cycle refrigerator. The CHNF system made it possible to measure the $I-V$ characteristics and electrical resistance in the range of temperatures $1.8 \mathrm{~K}<T<310 \mathrm{~K}$ and in magnetic fields with an induction $B$ as high as $8 \mathrm{~T}$. The used temperature controller (Lakeshore, model 331) made it possible to perform continuous scanning of the temperature with a rate of $0.1-1 \mathrm{~K} / \mathrm{min}$ or stabilize the temperature with an accuracy of $0.005 \mathrm{~K}$ during the course of scanning the magnetic field or during measurements of the $I-V$ characteristics. Thermometers based on gallium-arsenide diodes used in the temperature range $1.5-20 \mathrm{~K}$ were calibrated to an accuracy of $0.5 \mathrm{mK}$. The used devices for measurement of the voltage and current made it possible to determine the electrical resistance with an accuracy of greater than $0.1 \%$.

\section{RESULTS}

In this study, we measured the temperature dependences of the specific resistance under direct current, and of $\rho(T), \mathrm{MC}$, and the Hall effect insingle-crystal silicon doped with antimony $\mathrm{Si}: \mathrm{Sb}$. Measurements of the temperature dependence of the Hall effect made it possible to estimate the antimony concentration whose value was found to be $N_{\mathrm{Sb}} \approx 1 \times 10^{18} \mathrm{~cm}^{-3}$, which is much lower than the critical Mott concentration $N_{c}$ and the values of $N_{\mathrm{Sb}}$ in the samples studied previously [19]. This means that we can disregard the effects of weak localization and percolation in the experimental and theoretical analysis of the crossover in specific resistance in the studied temperature range.

Recent preliminary analysis [20] of the experimental data of measurements of the specific resistance for samples of $\mathrm{Si}: \mathrm{Sb}$ in the temperature range of 5-25 K and in magnetic fields as high as $8 \mathrm{~T}$ showed the following. In fields $B>1.5 \mathrm{~T}$, the magnetic-field dependence of the MC follows the trend $\ln \left[\rho(B) / \rho_{0}\right] \propto B^{2}$. An analysis of the dependences $\rho(T)$ indicated that, in $\mathrm{Si}: \mathrm{Sb}$ in the temperature range of 5-25 K, the VRH mode with the Mott mechanism takes place. The obtained estimates of the density of states $g_{0}$, the Mott temperature $T_{M}$, and the localization radius $a$ revealed the temperature dependence of the localization radius [20]. In the context of the Demishev-Pronin model [21] of hopping conduction (this model takes into account the presence of the spin-polarization mechanism and the mechanism of compression of the wave function for hopping conduction according to the Mott mechanism), the presence of two temperature regions, which are characterized by differing contribution of these mechanisms to $\rho(T)$, was established. It was established that spin-polarization transport is dominant in the range 5-11 K, while, in the range 11$20 \mathrm{~K}$, it is necessary to take into account both the contribution of spin-polarization transport and the contribution of the mechanism related to compression of the wave function in $\rho(T)$.

Figure 1 shows the experimental dependences $\rho(T)$ in the temperature range $2-25 \mathrm{~K}$; the dependences were measured with a small temperature step and careful stabilization (in the range of $\pm 1.5 \mathrm{mK}$ ) of the temperature at each experimental point. These measurements confirmed the previously observed [20] implementation of the VRH mode with the Mott mechanism at temperatures higher than $5 \mathrm{~K}$. As can be seen, in the case of cooling of the sample in the region 4.5-5.0 K, the transition from the Mott mode to the mode of hops to nearest neighbors (the NNH mode), which is observed in the temperature region 2.5$4.5 \mathrm{~K}$, occurs. The performed estimates of the conductivity activation energy for the $\mathrm{NNH}$ mode showed that these energies are $E_{N} \approx(17-18) k_{\mathrm{B}} \mathrm{J}\left(T_{N} \approx 17.6 \mathrm{~K}\right)$ in zero magnetic field and $E_{N} \approx 24.75 k_{\mathrm{B}} \mathrm{J}\left(T_{N} \approx\right.$ $24.75 \mathrm{~K})$ at $B=8 \mathrm{~T}$. For Si:Sb, estimation of the impurity localization radius from the expression $a=\hbar / \sqrt{2 m E_{i}}$ yielded the value $a \approx 3.3 \mathrm{~nm}$ (at an effective mass of $m=0.19 m_{0}$ and ionization energy of $E_{i}=0.036 \mathrm{eV}$ ).

At temperatures below $2.5 \mathrm{~K}$, there is a transition region, which is probably caused by transition to the 

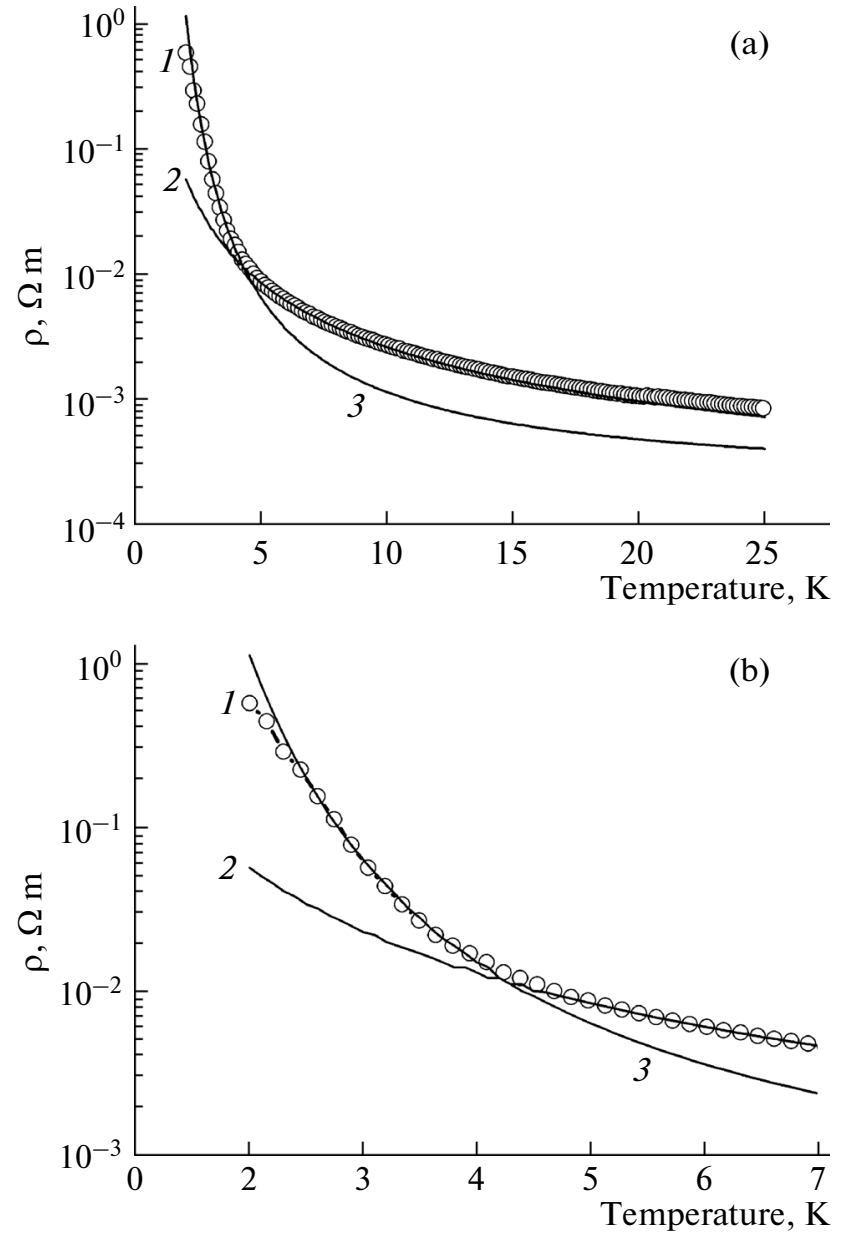

Fig. 1. Temperature dependence of the specific resistance $\rho(T)$ of a Si:Sb sample in the ranges of (a) $2-25 \mathrm{~K}$ and (b) 2-7 K: (1) experimental data, (2) results of calculations based on relation (1) for the Mott mechanism, and (3) results of calculations based on relation (1) for the NNH mode.

VRH mode with the Shklovskii-Efros mechanism. However, detailed analysis of this range requires additional studies.

In order to correctly use the models of crossovers (both those which already exist and that developed in this study, see below), it is necessary to determine the lattice type stipulated by the location of impurity atoms and also the percolation threshold for samples applicable in our experiments. In the theory of hopping conduction, problems related to percolation in a system of random lattice sites (chaotically distributed in space) play the most important role. In this case, the average number of lattice sites in the unit volume is assumed to be given (equal to the impurity concentration $N_{D}$ ), while the average distance between these sites is $r_{m}=\left(3 / 4 \pi N_{D}\right)^{1 / 3}$. For such a lattice, the percolation threshold $r_{c}$ (or, as it is also known, the percolation radius) is determined from the condition of the connectivity of two or more lattice sites, which form an infinite cluster [5], so that the value of $r_{c}$ depends only on the concentration of sites $N_{D}$. Typically, the threshold value $B_{c}$ is used, which has the meaning of the average number of bonds per site. The expression for the dimensionless percolation threshold in the three-dimensional case [5] is written as

$$
B_{c}=(4 \pi / 3) r_{c}^{3} N_{D} \text {. }
$$

The threshold value of the average number of bonds per site $B_{c}$ for a random lattice is typically determined numerically by solving the sphere problem via application of the Monte Carlo method. Numerous calculations yield values of $B_{c}$ in the range 2.65-2.7 [5]. Consequently, the percolation radius is given by $r_{c}=$ $(0.865 \pm 0.015) N_{D}^{-1 / 3}$. In this case, $r_{c}$ is always larger than the average distance between impurity atoms in a random lattice $r_{m}=0.62 N_{D}^{-1 / 3}$. At the concentration $N_{D}=N_{\mathrm{Sb}} \approx 10^{18} \mathrm{~cm}^{-3}$ in the samples we used, we have $r_{c}=8.64 \mathrm{~nm}$ and the average distance between the impurity atoms in the sample is $r_{m}=6.2 \mathrm{~nm}$. In this case, only the number of sites with the radius $r_{c}$ is important while their relative position (the lattice symmetry) is unimportant.

\section{MODEL AND DISCUSSION OF RESULTS}

Taking into account the fact that the aforementioned estimates are reasonable, we then developed a model of crossover from the VRH mode with the Mott mechanism to the NNH mode; this model is based on a simplified procedure for solving the percolation problem with the use of an interpolation expression for the density of states, fair for both modes. The suggested model is based on the following expression for the dimensionless concentration of sites

$$
N(\xi)=2 \int_{0}^{E_{\max }} r_{\max }^{3} g(E) d E,
$$

which satisfies the condition of connectivity at a hopping exponent (see (2)) smaller than a certain value $\xi$ $[5,9]$. Here, $E_{\max }$ and $r_{\max }$ are maximal values of the energy and the distance between lattice sites, which allow connectivity (see (5) and below), and $g(E)$ is the density of states. Equating expression (4) to the critical concentration in (3) for the percolation threshold $2 B_{c}$ (for the three-dimensional situation, the corresponding value is equal to $\sim 5.3$ ), we can obtain an equation for the critical value of the percolation exponent $\xi(T)$, which determines the electrical resistance. The dimensionless concentration $N(\xi)$ in (4) is determined as a product of the site concentration with hop energies $E<E_{\max }$ and the volume available for these sites. Such a procedure (used by Shklovskii and Efros [5] in relation to the Mott mechanism) for the state density dependent on energy is not at all strict and the condi- 
tion of connectivity should be considered for each energy value separately, which results in a complex integral equation [10]. We use the procedure described in [9], which makes it possible to considerably simplify the calculations.

The largest values of the energy and the distance between lattice sites, as allowed by the condition of connectivity in percolation theory [5] (see also formulas (4.30) in [22]) are determined by the formulas

$$
r_{\text {max }}=a \xi / 2, \quad E_{\max }=k_{\mathrm{B}} T \xi .
$$

Here, the critical value of the exponent $\xi$ is involved in the temperature dependence of the hopping resistance (2).

For the density of states, we use the interpolation expression which is fair for the case where the exponent $\rho$ in expression (1) for the resistivity approaches unity [15]. Rosenbaum et al. [12, 14, 15] described the models of crossovers from the Mott mechanism to the Shklovski-Efros mechanism and the NNH mode taking into account a variation in the density of states from its constant value for the Mott mechanism to the density of states $g(E) \propto|E|^{n}$ with $n>2$. According to [23], the exponent $n$ in the expression for $g(E)$ is related to the exponent $p$ in the three-dimensional VRH model (1) (see [5]) by the expression

$$
p=(n+1) /(n+4) \text {. }
$$

In this case, according to Rosenbaum [14], in order to describe the crossover at different combinations of $p$ values, it is possible to use the formula

$$
g(E)=\alpha E_{s g}^{n} \frac{\left(E / E_{s g}\right)^{n}}{1+\left(E / E_{s g}\right)^{n}}=g_{0} \frac{\varepsilon^{n}}{1+\varepsilon^{n}},
$$

where $g_{0}$ is the density of states in the absence of the Coulomb gap (at $n=0$ ). Here, the value of $n$ can be determined from relation (6) for the exponent $p$ in the VRH model. The values of $\alpha$ and $E_{s g}$ in (7) depend on $n$ and are constant at a fixed value of $n$. In the case $n=2$, the density of states coincides with (7) used in [12] for description of the crossover from the Mott mechanism to the Shklovskii-Efros mechanism.

Calculation of the integral (4), with (7) taken into account in the case where $n$ is an integer even positive number, yields the expression

$$
\begin{gathered}
N(\xi)=2 r_{\max } \alpha E_{s g}^{n+1} \int_{0}^{\varepsilon_{m}} \frac{\varepsilon^{n} d \varepsilon}{1+\varepsilon^{n}}=2 r_{\max } \alpha E_{s g}^{n+1} \\
\times\left[\varepsilon_{m}+\frac{1}{n} \sum_{k=0}^{(n / 2)-1} \ln \left(1+\varepsilon_{m}^{2}-2 \varepsilon_{m} \cos k_{n}\right) \cos k_{n}\right. \\
\left.-\frac{2}{n} \sum_{k=0}^{(n / 2)-1} \arctan \left(\frac{\varepsilon_{m}-\cos k_{n}}{\sin k_{n}}\right) \sin k_{n}\right]
\end{gathered}
$$

where $\varepsilon_{m}=\xi\left(k_{\mathrm{B}} T / E_{s g}\right), \cos k_{n}=\cos \left(\frac{2 k+1}{n} \pi\right)$, and $\sin k_{n}=\sin \left(\frac{2 k+1}{n} \pi\right)$. Equating (8) to the value $2 B_{c}=5.3$ and substituting expressions (5), we obtain, after transformations of the equation, which will be used for determination of the function $\xi(T)$ :

$$
\begin{gathered}
{\left[\xi \frac{T}{T^{*}}+\frac{1}{n} \sum_{k=0}^{(n / 2)-1} \ln \left(1+\left(\xi \frac{T}{T^{*}}\right)^{2}-2\left(\xi \frac{T}{T^{*}}\right) \cos k_{n}\right)\right.} \\
\left.\times \cos k_{n}-\frac{2}{n} \sum_{k=0}^{(n / 2)-1} \arctan \left(\frac{\xi\left(T / T^{*}\right)-\cos k_{n}}{\sin k_{n}}\right) \sin k_{n}\right] \\
=\frac{21.2}{g_{0}(a \xi)^{3} k_{\mathrm{B}} T},
\end{gathered}
$$

where $k_{\mathrm{B}} T^{*}=E_{s g}$. Taking into account the value of the Mott parameter $T_{M}$, we obtain the final equation

$$
\begin{aligned}
& \xi \frac{T}{T^{*}}+\frac{1}{n} \sum_{k=0}^{(n / 2)-1} \ln \left(1+\left(\frac{\xi T}{T^{*}}\right)^{2}-2\left(\frac{\xi T}{T^{*}}\right) \cos k_{n}\right) \cos k_{n} \\
& -\frac{2}{n} \sum_{k=0}^{(n / 2)-1} \arctan \left(\frac{\xi T}{T^{*} \sin k_{n}}-\cot k_{n}\right) \sin k_{n}=\frac{T_{m}}{T^{*} \xi^{3}},
\end{aligned}
$$

which provides the dependence $\xi(T)$ for a specified set of parameters $n, T^{*}$, and $T_{M}$ at $n \geq 4$. At an odd number $n$, the relation (10) changes its form and is represented by the expression

$$
\begin{gathered}
\xi \frac{T}{T^{*}}-\frac{1}{n} \ln \left(1+\left(\frac{\xi T}{T^{*}}\right)\right) \\
+\frac{1}{n} \sum_{k=0}^{(n / 2)-3 / 2} \ln \left(1+\left(\frac{\xi T}{T^{*}}\right)^{2}-2\left(\frac{\xi T}{T^{*}}\right) \cos k_{n}\right) \cos k_{n} \\
-\frac{2}{n} \sum_{k=0}^{(n / 2)-3 / 2} \arctan \left(\frac{\xi T}{T^{*} \sin k_{n}}-\cot k_{n}\right) \sin k_{n}=\frac{T_{M}}{T^{*} \xi^{3}},
\end{gathered}
$$

for $n \leq 5$.

Comparison of the results of calculations performed on the basis of relations (2), (10), and (11) with the experimental dependences in Fig. 1 showed that, for the previously obtained parameters $T_{M}=15240 \mathrm{~K}$ [16] and $T^{*}=T_{N}=(17-18) \mathrm{K}$, the developed model of crossover adequately describes the experimental data: at $n=0$ (Mott mechanism; absence of the Coulomb gap), coincidence was obtained in the region 4-25 K (Fig. 2a); for $n=6$ and larger, there is coincidence in the region $2.5-5.0 \mathrm{~K}$ (the NNH mode).

Thus, using the developed model, we may adequately describe the temperature dependence $\rho(T)$ as hopping conduction is varied from the VRH mode for 

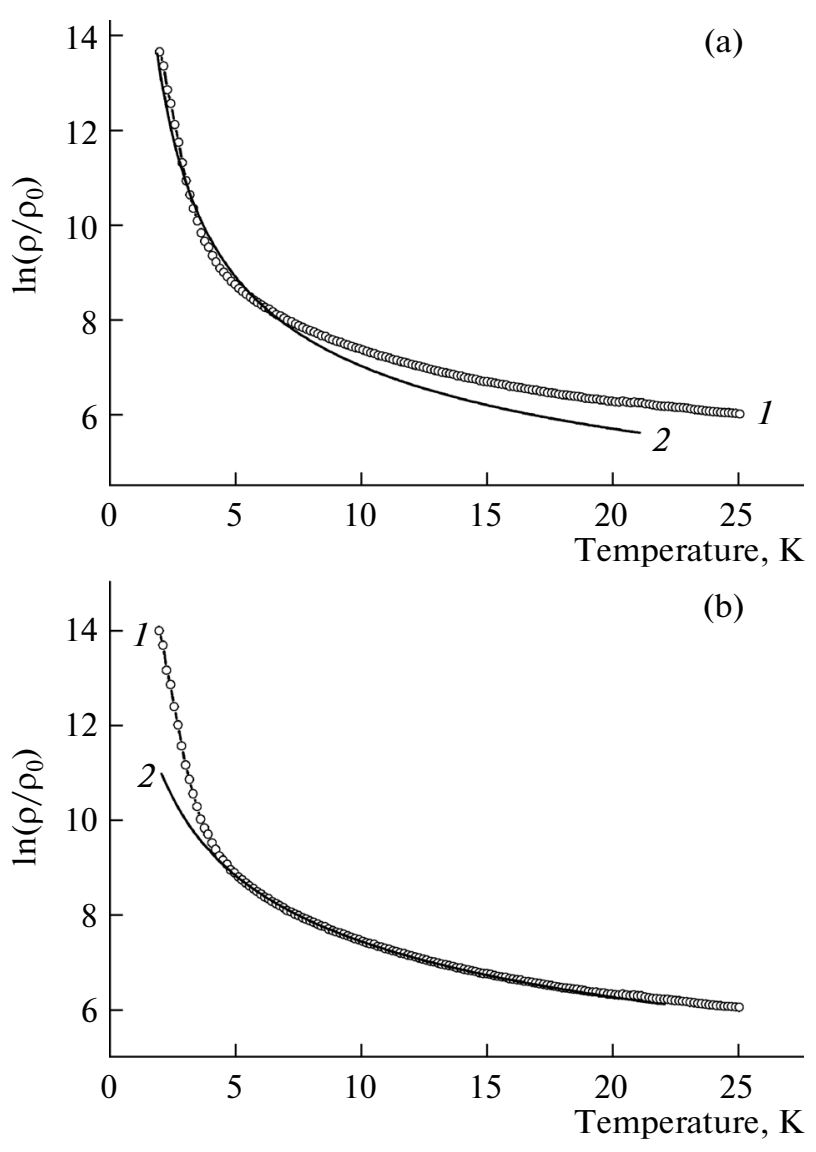

Fig. 2. Experimental (1) and calculated (2) temperature dependences of the normalized resistivity $\left(\rho_{0}\right.$ is the preexponential factor in (1) as determined from the experimental data) of a Si:Sb sample for the NNH mode, $n=7$ (a) and the Mott mechanism, $n=0$ (b).

the Mott mechanism to the NNH mode using the corresponding parameters of these modes at a fixed value of exponent $n$. The value of $n$ does not introduce any doubts for the Mott mechanism $(n=0)$ and for the Shklovskii-Efros mechanism $(n=2)$. The choice is not quite unique for the NNH mode. It follows from expression (6) that the value of $n$ is bound to be much larger than unity. If we intend to determine the optimal (or minimal) choice of the value of $n$ for the NNH mode, we may use the connection of the function $\xi \propto$ $\left(T^{*} / T\right)^{p}$ with the exponent $p$ for the calculation of $\rho$ from the obtained model using the expression

$$
p=-d \log \xi / d \log T=-(T / \xi) d \xi / d T,
$$

reported in publication [9]. It follows from (12) that, for $n=0$, the exponent $p$ is exactly equal to 0.25 in the entire temperature range (as in relation (1) for the Mott mechanism), while $p$ depends on temperature for other values of $n$. This dependence estimated from expressions (10) and (11) for various values of $n$ is represented in Fig. 3.

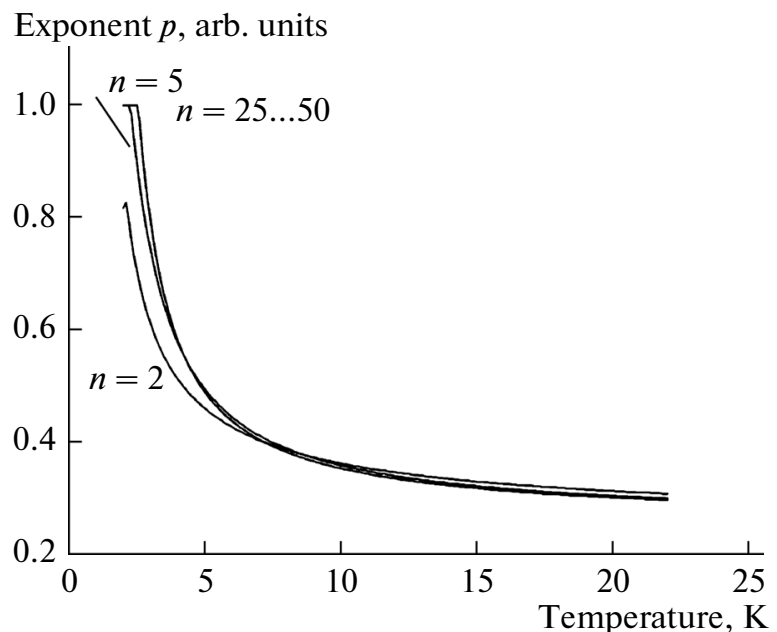

Fig. 3. Temperature dependences of the exponent $p$ in relation (1) as obtained using expressions (10)-(12) at $n \geq 2$.

The obtained dependences are represented for clarity at fixed values of $n$ for the entire range of temperatures. However, the curves for $n=5-50$ reflect the adequacy of the model in the region $T<4 \mathrm{~K}$ and make it possible to conclude that, in order to attain values of exponent $p$ close to unity, it is sufficient to use $n_{\min }=5$; also, there is no point in increasing $n$ to values larger than 25 (since it is clear that the curves for $n=25$ and $n=50$ coincide Fig. 3 ). It first of all follows from Fig. 3 that, at $T>5 \mathrm{~K}$, the NNH mode is clearly not observed in our experiments since the exponent $p$ ceases to depend on $n$ at all, which is precisely indicative of the predominance of the Mott mechanism under the above conditions.

The developed model makes it possible to show that the theoretical range of crossover from the VRH mode to the NNH mode should be in the range 3-7 K. The experiment fixes this transition at a temperature of $4.5 \mathrm{~K}$ exactly. It is worth noting that such a spread is observed for all known models of crossovers, which represents its main disadvantage at present.

\section{CONCLUSIONS}

The performed detailed analysis of the obtained experimental results for the temperature dependences of the specific resistance in the range $2-25 \mathrm{~K}$ for silicon doped with antimony showed that, as a result of cooling to a temperature lower than $T=4.5 \mathrm{~K}$, the temperature crossover from the Mott mechanism to the mode of hopping conduction via the nearest neighbors ( $\mathrm{NNH}$ ), while, at $T<2.5 \mathrm{~K}$, the transition to the Shklovskii-Efros mechanism possibly takes place. We suggested a model describing the temperature crossover from the Mott mechanism to the mechanism of hopping conduction via nearest neighbors; the model is based on a simplified solution of the percolation problem with the use of an interpolation 
expression for the density of states (this expression is valid for both modes). We obtained equations for determination of the critical value of the exponent in the temperature dependence of the hopping resistance. The performed estimations showed that it is possible to attain satisfactory agreement between theoretical and experimental crossovers with the use of an optimal number of adjustable parameters.

\section{ACKNOWLEDGMENTS}

This study was partly supported by the State Program of Scientific Studies "Functional and Compositional Materials, Nanomaterials" of the Republic of Belarus 2013-2014 (project no. 2.4.10).

\section{REFERENCES}

1. Y. L. Zhao, W. M. Lv, Z. Q. Liu, S. W. Zeng, M. Motapothula, S. Dhar, Ariando, Q. Wang, and T. Venkatesan, AIP Adv. 2, 012129 (2012).

2. A. Narjis, A. E. Kaaouachi, A. Sybous, L. Limouny, S. Dlimi, A. Aboudihab, J. Hemine, R. Abdia, and G. Biskupski, J. Mod. Phys. 3, 517 (2012).

3. N. F. Mott, Metal-Insulator Transition, 2nd ed. (Taylor and Francis, London, 1990).

4. P. P. Edwards and M. J. Sienko, Phys. Rev. B 17, 2575 (1978).

5. B. I. Shklovskii and A. L. Efros, Electronic Properties of Doped Semiconductors (Springer, New York, 1984; Moscow, Nauka, 1979).

6. N. V. Agrinskaya and A. N. Aleshin, Sov. Phys. Solid State 31, 336 (1989).
7. A. Aharony, Y. Zhang, and M. R. Sarachik, Phys. Rev. Lett. 68, 3900 (1992).

8. I. S. Shlimak, Semiconductors 27, 1069 (1993).

9. N. V. Agrunskaya and V. I. Kozub, J. Exp. Theor. Phys. 89, 1125 (1999).

10. Y. Meir, Phys. Rev. Lett. 77, 5265 (1996).

11. N. van Lien, Phys. Lett. A 207, 379 (1995).

12. R. Rosenbaum, N. van Lien, M. R. Graham, and M. Witcomb, J. Phys.: Condens. Matter 9, 6247 (1997).

13. H. Fritzsche and M. Guevas, Phys. Rev. 119, 1238 (1960); R. Ray, and H. Fan, Phys. Rev. 121, 768 (1961).

14. N. van Lien and R. Rosenbaum, J. Phys.: Condens. Matter 10, 6083 (1998).

15. N. van Lien and R. Rosenbaum, Phys. Rev. B 56, 14960 (1997).

16. A. G. Zabrodskii and K. N. Zinov'eva, Sov. Phys. JETP 59, 366 (1984).

17. A. G. Zabrodskii, Phys. Usp. 41, 722 (1998).

18. N. V. Agrinskaya and V. I. Kozub, J. Exp. Theor. Phys. 79, 466 (1994).

19. A. Fujimoto, H. Kobori, T. Ohyama, S. Ishida, K. Satoh, T. Kusaka, and Y. Kakehi, Physica B 324, 1 (2002).

20. A. Fedotov, S. Prischepa, A. Danilyuk, I. Svito, and P. Zukowski, Acta Phys. Polon. A 125, 1271 (2014).

21. S. V. Demishev and A. A. Pronin, Phys. Solid State 48, 1363 (2006).

22. B. I. Shklovskii and A. L. Efros, Sov. Phys. Usp. 18, 845 (1975).

23. E. M. Hamilton, Philos. Mag. 26, 1043 (1972).

Translated by A. Spitsyn 